\title{
Integrating Spatial-Temporal Risk Factors for an Ambulance Allocation Strategy: A Case Study in Bangkok
}

\author{
Pakinai Chaicharoenwut ${ }^{1}$, Jarotwan Koiwanit ${ }^{1}$, Pannapa Changpetch $^{2}$, Sirisuda Buatongkue $^{3}$, Chumpol Yuangyai $^{1 *}$ \\ ${ }^{1}$ Industrial Engineering Department, Faculty of Engineering, King Mongkut's Institute of Technology Ladkrabang, Bangkok, Thailand \\ ${ }^{2}$ Mathematical Science Department, Bentley University, Massachusetts, United States \\ ${ }^{3}$ Information Technology Department, Faculty of Science and Arts, Burapha University, Chanthaburi, Thailand
}

\begin{abstract}
Dedicated emergency medical services (EMS) are important to patients' chances of survival. In particular, the quicker such services arrive at the scene of an incident, the higher the survival rate. Therefore, the management of ambulance bases is an essential aspect of emergency medical services. Further, the locations of ambulance bases are determined based on patient demand. However, in practice, many elements should be taken into account in a risk assessment of given areas within a locale. Specifically, each area should be assessed for the number and severity of accidents that ordinarily take place there, the number and size of the public events it hosts, its population density, and the number of elderly people resident. In this study, we use a spatial-temporal approach to integrate those factors into a risk assessment of areas relative to each other in a locale. Based on this risk assessment, we determine the optimal locations for ambulance bases in order to minimize response time. We validate our approach using Bangkok as a case study.
\end{abstract}

\section{Introduction}

Emergency medical services (EMS) are crucial to health management [1]. Their primary purpose is to provide care to injured people at the scene of an accident or other incident requiring immediate care by providing primary care and then getting them to a hospital as fast as possible. Most EMS system planners draw on experience and information pertaining to the historical accidents per area to determine where to locate ambulance bases. However, this traditional approach is not optimal in terms of minimizing expenses, i.e., more bases than are necessary for a given area are built. Nor is this approach optimal in terms of minimizing response time, i.e., injured people wait longer than they would under another design.

For example, in 2014-2017 in Bangkok, Thailand, the EMS took longer than 10 minutes to reach the scene in $99 \%$ of incidents (Emergency Medical Information System: ITEMS 2559), which exceeds the standard time of 8 minutes [2]. A delay of this nature may have an adverse effect on victims' chances of surviving.

The allocation problem in regard to emergency base location has generally been considered using a covering model. Introduced in 1971 by Toregas et al. [3], the first covering model referred to as the Location Set Covering Problem (LSCP) had the goal of establishing the minimum number of ambulance bases needed to cover a service area. Variants include the Location Set Covering Problem (LSCP) [3] and the Maximal Covering Location Problem (MCLP) [4]. Most of the models used to determine where to locate bases take only patient demand into account. Yet, several kinds of information should be considered concurrently. In this study, therefore, we integrate multiple kinds of spatial-temporal information such as the number and the severity of accidents per area, population density, the number of elderly living in each area, and the number and size of the public events that take place there.

Designed to consider factors involving space and time simultaneously, spatial-temporal analysis is usually applied to a geographic information system (GIS) in order to define areas of relative risk. The higher the risk area, the greater the number of accidents that take place there. On this basis, we undertake accessibility risk identification in order to identify high-, medium-, and low-risk areas in order to allocate the number of ambulances per area more effectively than is possible under the standard approach.

\section{Literature Review}

In this section, we present accounts of the research relevant to our study, the coverage models, a method for accessibility risk identification, and a spatial-temporal analysis of accessibility risk.

\subsection{A covering models}

Determining the ambulance bases for an emergency medical service system is very important. In studies over published over the last 40 years, researchers have proposed several methods of management. In particular, the covering model is often used to determine ambulance bases, and mathematical versions of the covering model are used in public service planning to cover most areas of service [5]. Overall, the covering model has been applied in many kinds of organizations, such as transport planning, fire stations, and emergency medical services including allocating ambulances. In terms of ambulance allocation, the goal is to meet (cover) the needs of victims

\footnotetext{
* Corresponding author: chumpol.yuangyai@gmail.com
} 
at the scene by ensuring that ambulances reach them quickly and thereby optimizing chances of survival. The Location Set Covering Problem (LSCP) model [3] was used to determine the minimum number of ambulance bases needed for a given service area. However, in this basic model there are no restrictions on the resources available to establish and operate bases. In 1974, Church and ReVelle [4] modified this basic covering model in their Maximal Covering Location Problem (MCLP) by adding demand (i.e., meeting patients' needs) to the covering model. In this model, the goal is to cover most of the demand using a limited number of ambulance base. Each of these models has been applied at both the operational level and the strategic planning level of ambulance base allocation.

In most of the relevant research, patient demand constitutes the principal or even the only information taken into account in determining where to locate ambulance bases. In practice, numerous factors affect ambulance allocation, such as the number and severity of accidents in an area, population density, the number of elderly, public events, and whether or not the area is likely to suffer a natural disaster. Thus, in the present research, we integrate these risk factors into the covering model proposed by Nilsang et al. [6] with the overall purpose of improving response time.

\subsection{Determining risk factors to account for in determining ambulance base allocation}

In this section, we discuss the spatial-temporal risk factors that should be taken into account in determining the locations of ambulance bases. These factors include:

- Number of accidents: This aspect, which refers to the demand for emergency medical services, is the principal factor considered in the covering model [7].

- Severity of accidents: Accidents vary greatly in terms of severity, such that emergency medical services should be capable of adapting accordingly. [8].

- Population density: When the population density of an area is high, there is likely to be a correspondingly high need for emergency services. It is, therefore, necessary to locate an ambulance base or bases in such areas.

- Number of elderly: Elderly people have a high need for medical services. Therefore, it is necessary to locate an ambulance base or bases in areas where a high number of elderly people live [9].

- Number and size of public events: Public events that attract a large number of people, whether national sporting events, University graduation ceremonies, or music festivals, present risk such that emergency medical services should be readily available. Therefore, an ambulance base or bases should be located in areas where such events take place [10].

We evaluate all 5 of these risk factors in our model, and we describe how we assess risk level in Section 2.3.

\subsection{Risk assessment and analysis of spatial-temporal risk factors}

The analysis of spatial-temporal risk factors is an aspect of the Geographic Information System (GIS) in which location and time are considered concurrently. This approach has been used in several areas: anomaly detection $46 \%$ on disaster management, $27 \%$ in traffic management $14 \%$ inference place $13 \%$ health management 5\% which information from Twitter [11].

In this study, we assess multiple spatial-temporal risk factors to determine the level of risk (risk values) in each area. We use risk values to determine the optimal places to locate ambulance bases. As we will show, our model is designed to take all risks affecting ambulance base allocation into account.

\section{Overview of Our Integrated Model}

In this section, we present our new covering model in which risk values instead of patient demand values are used to determine the locations of ambulance bases.

\subsection{Assessment of spatial-temporal risk factors}

Our assessment of spatial risk factors includes the number of accidents in the area, the severity of the accidents, the population density, the number of elderly people living in the area, and the number and size of public events that take place there. The criteria for risk assessment are presented in Appendix 1. The assessment of the risk value of each factor is presented in Table 1 .

To determine the spatial risk of each factor, the spatial risk of each factor used to obtain the spatial risk totally applied to a covering model (Equation 1):

$$
R_{i}=\prod_{h=1}^{5}\left(L_{i h} \times I_{i h}\right)
$$

The model is described as follows: $R_{i}$ is the risk value for area $i ; L_{i h}$ is the likelihood of risk factor $h$ for area $i ; I_{i h}$ is the impact of risk factor $h$ for area $i ; i$ is the index of the area; and $h$ is the index of the risk factor.

In a covering model, the total risk of each area is used to determine the ambulance bases needed to cover the maximum risk and set the range of the total risk values for each area. The areas with the lowest risk are shown in purple, and the highest-risk areas are shown in red.

\subsection{Covering model with risk factors}

Next, the risk assessment of each study area is used with a mathematical model to determine the ambulance base allocation. We integrate spatial-temporal risk values into the MCLP model. In addition, we incorporate the concept of location-forced constraints as proposed by Nilsang et al. [6] into our model. An MCLP model relies on the following equations (2-7): 


$$
\text { Maximize } \quad \sum_{i=1} R_{i} y_{i}
$$

Constraints

$$
\begin{aligned}
& \sum_{j \in W_{i}} x_{j} \geq y_{i}, i \in V \\
& \sum_{j \in W_{i}} x_{j} \leq P \\
& \beta_{j} \leq x_{j} \quad j \in W \\
& x_{j} \in\{0,1\}, j \in W \\
& y_{i} \in\{0,1\}, i \in V
\end{aligned}
$$

\section{Notations:}

$$
\begin{aligned}
V & =\text { Set of area points } \\
W & =\text { Set of potential base locations } \\
i & =\text { Index of area points, } i \in V \\
j & =\text { Index of potential base locations, } j \in W \\
R_{i} & =\text { Risk value in area point } i \\
P & =\text { Total potential base location }
\end{aligned}
$$$$
\beta_{j}=\left\{\begin{array}{l}
1 \text { if base location is location-forced } j \\
0 \text { otherwise }
\end{array}\right.
$$$$
x_{j}=\left\{\begin{array}{l}
1 \text { if base location is selected at site } j \\
0 \text { otherwise }
\end{array}\right.
$$$$
y_{i}=\left\{\begin{array}{c}
1 \text { if area point } \mathrm{i} \text { is covered at least onec } \\
0 \text { otherwise }
\end{array}\right.
$$

Objective (2) is to maximize the risk value coverage. Constraint (3) guarantees that an area is covered only if at least one ambulance base is located within the distance standard. Constraint (4) guarantees that the total number of potential base locations is less than P. Constraint (5) guarantees that the control location $j$ is selected. Constraints (6) and (7) $x_{j}$ and $y_{i}$ together constitute the binary decision variable.

\section{Case Study}

We demonstrate our proposed approach to ambulance base allocation and spatial risk assessment using the EMS in Bangkok, Thailand, as a case study. The data collection and parameters are described next.

\subsection{Description of the EMS in Bangkok}

The capital of Thailand, Bangkok has an area of 1,568.74 kilometers ${ }^{2}$ and a population of 5,686,646, which means that it has the highest population density in the country. There are 43,959 elderly people living in each area of Bangkok, who are at a high risk of needing emergency medical services. As the country's capital, Bangkok has a large convention center and a national stadium such that the city hosts many public events. When there is an emergency situation at events in particular, it is important that extensive and appropriate medical services be readily available.

\subsection{Data collection and risk assessment}

For this case study, we collected data pertaining to the number of severity of accidents in the Bangkok city area in January 2018. We obtained data on population density and the number of elderly people living in the city from the Ministry of the Interior, and public event information from the Internet. Next, we divided Bangkok into 56 blocks of $36 \mathrm{~km}^{2}(6 \times 6 \mathrm{~km})$ to use as a basis for decision making. We used this information to assess the risk of each area in our covering model to determine emergency base allocation. The color temperature is divided into 10 levels according to the risk used in decision making, parking ambulance. The purple area represents low risk $\left(R_{i} \leq 99\right)$, and the red area represents high risk ( $\left.R_{i} \geq 1,000\right)$. We used the proposed coverage model to find the ambulance location.

\section{Results}

We compared the results of the MCLP-Demand and MCLP-Risk values in the MCLP model for Bangkok, which, as stated, we divided into 56 blocks for which we represented risk using a color scheme (Figure 1). A black circle symbol is used to represent the ambulance bases. The result is that the MCLP-Risk value determines the number of ambulance bases which cover more than MCLP-demand shown in Figure 1.

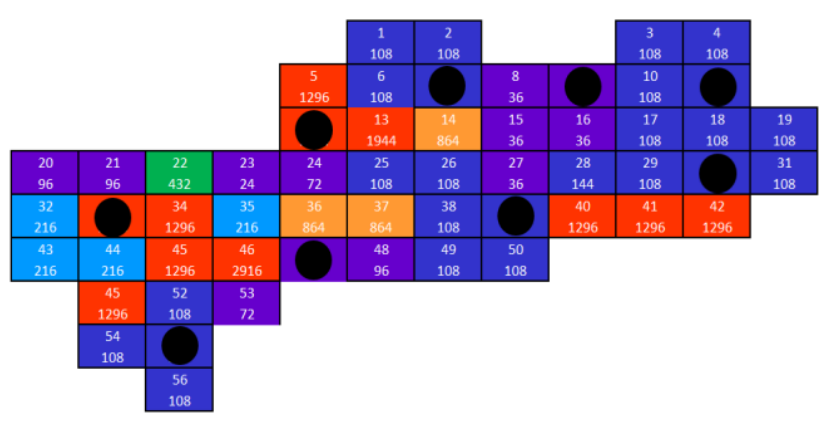

(a) MCLP - Risk Value

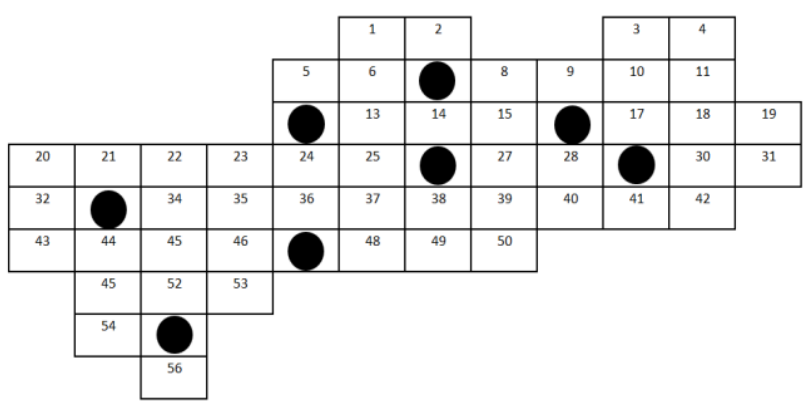

(b) MCLP - Demand

Fig. 1. Ambulance base locations determined using the MCLP model for 56 areas of Bangkok.

Our analysis shows a difference in the results in the MCLP model. The MCLP-Risk value model assigns 9 ambulance bases to cover the risk in the Bangkok area, 
whereas the MCLP-Demand model assigns 8 ambulance, which do not cover the entire Bangkok area. Our model relies on these 5 risk factors in a covering model together with the color scheme for decision making. On the basis of our model compared with the standard model, the ambulance base allocation has a greater coverage area and victims are admitted to hospital more quickly.

\section{Conclusions and Discussion}

We have presented a spatial-temporal model for identifying risk factors that affect the effectiveness of emergency medical systems. We found that 5 factors should be considered in allocating ambulance base locations: the number and severity of the accidents per area, its population density, the number of elderly people living there, and the number and size of the public events held there. We bring all of these risk factors to bear on assessing the risk-value of each area in terms of providing emergency medical services. We defined the criteria for determining the risk value of each area and presented this information using a color scheme to indicate risk levels in a given area. Next, we integrated the risk values into the standard MCLP model to allocate ambulance bases in order to cover all the high-risk areas. We also validated our model by applying it to Bangkok.

In future research, we plan to include information pertaining to the number and size of public events from social media. Further, we plan to add other spatialtemporal risk factors such as the amount of rainfall and conflagration incidents in determining where to allocate ambulance bases in order to improve the performance of emergency medical systems.

\section{References}

1. Lim CS, Mamat R, Braunl T (2011) Impact of ambulance dispatch policies on performance of emergency medical services. IEEE Trans Intell Transp Syst 12:624-632. doi: 10.1109/TITS.2010.2101063
2. De Maio VJ, Stiell IG, Wells GA, Spaite DW (2003) Optimal defibrillation response intervals for maximum out-of-hospital cardiac arrest survival rates. Ann Emerg Med 42:242-250. doi: 10.1067/mem.2003.266

3. Toregas, C., et al., The location of emergency service facilities. Operations research, 1971. 19(6): p. 1363-1373.

4. Church, R. and C. ReVelle. The maximal covering location problem. in Papers of the Regional Science Association. 1974. Springer.

5. Farahani RZ, Asgari N, Heidari N, et al. (2012) Covering problems in facility location: A review. Comput Ind Eng 62:368-407. doi: 10.1016/j.cie.2011.08.020

6. Nilsang S, Yuangyai C, Janjarassuk, U, Cheng $\mathrm{CY}$, Locating an ambulance base by using social media: A case study in Bangkok. Annals of Operations. Research In process of public.

7. Li X, Zhao Z, Zhu X, Wyatt T (2011) Covering models and optimization techniques for emergency response facility location and planning: A review. Math Methods Oper Res 74:281-310. doi: 10.1007/s00186-011-0363-4

8. Ratanavaraha V, Suangka S (2014) Impacts of accident severity factors and loss values of crashes on expressways in Thailand. IATSS Res 37:130-136. doi: 10.1016/j.iatssr.2013.07.001

9. Peacock PJ, Peacock JL (2006) Emergency call work-load, deprivation and population density: An investigation into ambulance services across England. J Public Health (Bangkok) 28:111-115. doi: 10.1093/pubmed/fdi079

10. Laylavi F, Rajabifard A, Kalantari M (2017) Event relatedness assessment of Twitter messages for emergency response. Inf Process Manag 53:266-280. doi:

10.1016/j.ipm.2016.09.002

11. Steiger E, de Albuquerque JP, Zipf A (2015) An advanced systematic literature review on spatiotemporal analyses of Twitter data. Trans GIS 19:809-834. doi: 10.1111/tgis. 12132

Appendix 1. Assessment of Risk Values

\begin{tabular}{|c|c|c|c|c|c|c|}
\hline \multirow{2}{*}{ Factor } & \multicolumn{3}{|c|}{ Likelihood } & \multicolumn{3}{|c|}{ Impact } \\
\hline & 1 (Low) & 2 (Medium) & 3 (High) & 1 (Low) & 2 (Medium) & 3 (High) \\
\hline $\begin{array}{c}\text { Frequency of } \\
\text { accidents }\end{array}$ & $\begin{array}{c}0-2 \\
\text { days/week }\end{array}$ & $\begin{array}{c}3-5 \\
\text { days/week } \\
\end{array}$ & $\begin{array}{c}5-7 \\
\text { days/week }\end{array}$ & $<3$ times/day & 3-6 times/day & $>6$ times/day \\
\hline $\begin{array}{c}\text { Severity of } \\
\text { accidents }\end{array}$ & $\begin{array}{c}<3 \\
\text { times/day }\end{array}$ & $3-5$ times/day & $\begin{array}{c}>5 \\
\text { times/day }\end{array}$ & Small accidents & Medium accidents & Big accidents \\
\hline Population density & \multicolumn{3}{|c|}{ assign $=$ Low } & $<50,000$ units & $\begin{array}{c}50,000-100,000 \\
\text { units }\end{array}$ & $>100,000$ units \\
\hline Number of elderly & \multicolumn{3}{|c|}{ assign $=$ Low } & $50-500$ units & 501-1,000 units & $>1,000$ units \\
\hline $\begin{array}{c}\text { Number of } \\
\text { Public events }\end{array}$ & $\begin{array}{c}0-2 \\
\text { days/week }\end{array}$ & $\begin{array}{c}3-5 \\
\text { days/week }\end{array}$ & $\begin{array}{c}5-7 \\
\text { days/week }\end{array}$ & $<2,000$ units & $2,001-5,000$ units & $>5,000$ units \\
\hline
\end{tabular}

